\title{
FLARES IN T TAURI STARS
}




\section{Flares in T Tauri Stars}

Gösta F. Gahm

Stockholm Observatory, S-133 36 Saltsjöbaden, Sweden

\section{Introduction}

This review concerns rapid variability, on time-scales of a few hours or less, occurring on T Tauri stars (TTS). There are several recent reviews on observed properties of TTS (e.g. Appenzeller \& Mundt 1989, Bertout 1989, Gahm 1990a, Kuhi \& Cram 1989), some with more emphasis on "flare-like" activity (Feigelson et al. 1991, Gahm 1990b, Montmerle 1991, Montmerle et al. 1993), and the ambition below has been to summarize more recent work. We will also give a compilation of published works on observations of optical variability on these time-scales and a related statistical overview. Some early, very interesting and extensive studies of this kind were made here at the Sonneberg Observatory, where for instance Götz \& Wenzel (1967) concluded that the light-curve of RW Aurigae contains several components: quasi-periodic fluctuations over days, 'waves' over several hours, rapid outbursts with symmetric light-curves, rapid outbursts with asymmetric (flare-like) light-curves (see also Fürtig \& Wenzel 1964) and small fluctuations with very small amplitudes, which they postulated could be due to changes in the emission line fluxes. Modern photometric results of higher sensitivity and time-resolution confirm the existence of these different types of rapid variations in classical TTS (CTTS), having strong emission (lines and continuous, so called veiling) superimposed on the photospheric absorption line spectrum. As described in the subsequent chapters there are new concepts for the interpretation of the short-lived fluctuations. Concerning the long-term quasi-periodic variations we can now usually relate them to the stellar rotational period (bright or dark spots), let be that RW Aur still is an uncertain case. There are other slow changes most likely related to variable circumstellar extinction in circumstellar dust in the line-of-sight to the star. The long-term optical changes were most recently discussed by Herbst et al. (1994).

\section{X-ray bursts}

A number of X-ray bursts were observed with Einstein (see the reviews above). Some extremely energetic events were recorded, the most extreme one on the 
WTTS doublet ROX 20 with a peak luminosity of $10^{32} \mathrm{erg} \mathrm{s}^{-1}$ and a total Xray energy of $E_{x} \geq 10^{35} \mathrm{ergs}$ (Montmerle et al. 1983). An even more powerful burst on $\mathrm{LkH} \alpha 92$ with $510^{32} \mathrm{erg} \mathrm{s}^{-1}$ (peak) and $E_{x}=410^{36}$ ergs was detected by ROSAT (Preibisch et al. 1993) and the spectrum of what could be a TTS burst in the $\rho$ Oph region was traced to energies above $10 \mathrm{keV}$ by Koyama et al. (1992) from Ginga observations. The X-ray bursts are flare-like with short risetimes and exponential declines and they last for a few hours. From spectrally resolved events temperatures of a few $10^{7} \mathrm{~K}$ are inferred (model dependent) and if the X-ray emitting volumes are single loops rooted at the stellar surface, then they typically extend to up to about 1 stellar radius. These results are summarized and compared to corresponding events on the Sun and flare stars in Table 1, most of which is based on Preibisch et al. (1993), which should be consulted for references on ranges in the values - not given here. Data for post-T Tauri candidates (PTTS), older than the TTS but presumably still in contraction, come from Tagliaferri et al. (1988, HD 560B) and Pakull (1981, AB Dor: in parenthesis).

It has always been assumed that these X-ray bursts in fact are flares, similar to what is seen on the Sun and flare stars but much more powerful. In view of recent theoretical developments concerning possible interactions between a circumstellar disk and the star, one must also ask whether hot transient phases can develop from inhomogeneous mass accretion or cometary bodies flying into a stellar wind and disintegrating. On the basis of models (Camenzind 1990, Königl 1991; see also Ushida \& Shibata 1984, 1985 and Bertout et al. 1988) with accretion along magnetic loops connecting a circumstellar disk and the star, Gullbring (1994) showed, assuming inhomogeneous mass accretion, that X-rays will be produced if the optical depth in the plasma of infalling blobs is small. However, the temperature is determined by the potential energy extracted from the almost free falling plasma and can only reach a few $10^{6} \mathrm{~K}$. Hence, the hotter, strong $\mathrm{X}$-ray bursts are most likely connected to magnetic surface activity and not to accretion. This does not exclude the presence of $\mathrm{X}$-ray events generated by accretion. Strong $X$-ray flaring may be a rather rare phenomenon, however. Gagné \& Caillault (1995) have made a detailed examination of all the Einstein IPC and HRC fields in the region centred at the Orion nebula. From 45 hours of time-resolved observations only $2 \mathrm{X}$-ray flares with fluxes $\geq 2.510^{30} \mathrm{erg} \mathrm{s}^{-1}$ were found among the over $200 \mathrm{X}$-ray sources. $1 / 4$ th of the sources vary on time-scales of 1 day to 1 year.

\section{Radio bursts}

Radio emission at cm wavelengths has been detected from several TTS and many sources are known to vary with time. This radiation can be thermal and arise in massive stellar winds (Panagia \& Felli 1975) or non-thermal. The CTTS have radio spectra consistent with thermal emission while for the WTTS the evidence of sources of gyrosynchrotron radiation is increasing: First, the brightness temperatures derived are high, $10^{7.5}-10^{9} \mathrm{~K}$, much higher than those inferred from 
Table 1. X-ray flare characteristics

\begin{tabular}{lccc}
\hline Object & $\begin{array}{c}E_{x} \\
\left(10^{30} \mathrm{erg}\right)\end{array}$ & $\begin{array}{c}T \\
\left(10^{6} \mathrm{~K}\right)\end{array}$ & $\begin{array}{c}l \\
\left(10^{10} \mathrm{~cm}\right)\end{array}$ \\
\hline Sun, 3b flare & 10 & 10 & 1 \\
dMe (EV Lac) & $10^{4}$ & 30 & 2 \\
TTS & $10^{4}$ & 20 & 2 \\
ROX 20 & $10^{6}$ & 15 & 10 \\
LkH $\alpha$ 92 & $410^{6}$ & 50 & 8 \\
PTTS & $2 \cdot 10^{4}\left(4 \cdot 10^{6}\right)$ & 50 & \\
\hline
\end{tabular}

$\mathrm{X}$-ray data (Phillips et al.J1991). Second, the spectral index is flat or negative in many stars as was pointed out already by Bieging et al. (1984) and discussed by e.g. Stine et al. (1988). The VLA and VLBI studies made so far show that for some WTTS, the sources are very extended. Third, circular polarisation is present in many stars (O'Neal et al. 1990, Skinner 1993).

Considerable flux changes occur on time-scales of several hours (see e.g. White et al. 1992). Hence, the radio characteristics provide the best evidence of magnetic activity in WTTS. It is not straightforward to model the flares. Efforts in this direction have given magnetic field strengths of $B<1$ kGauss and single loop lengths of one up to $\sim 15$ stellar radii as envisioned by e.g. Montmerle \& André (1988). It should be pointed out that a lot of attention has been given to the most extreme cases DoAr 21 and HD 283447, which may be peculiar in the WTTS group.

The first simultaneous multi-frequency observations of TTS start to appear. Feigelson et al. (1994) observed what could be the declining part of a radio flare on (again) HD 283447 over a few hours when the X-ray, optical and emission line fluxes $(\mathrm{H} \alpha, \mathrm{Mg} \mathrm{II})$ stayed constant. In this paper several possible origins of TTS activity are discussed, including solar type activity, star-disk interactions, magnetic fields in the disk and binarity. More studies of this type are under way. Finally we note that a rapid decrease in the radio flux of T Tau (S) (a possible protostar?) was discovered by Phillips et al. (1993).

\section{Short-term optical variability}

Optical monitoring of TTS has a long history. In the review by Gahm (1990b) it was pointed out that: $1 . U$-band 'flaring' with changes by more than $20 \%$ in flux is rare; 2. CTTS most often show symmetric rather than asymmetric (flare-like) light-curves, like observed for RW Aur by Götz \& Wenzel (see Chapter 1); 3. Assuming black-body radiation, the events are cool $\sim 7000 \mathrm{~K}$, different from flares on the Sun and flare stars; 4 . These flares may originate from inhomogeneous mass accretion rather than from magnetic surface activity. More published results have been added to the data bank since that time and we can now make an overview of short-term variability of the stars sorted in CTTS and WTTS in 
Table 2. Stars in the data bank

\section{CTTS}

Taurus: RY, T, DF, DG, DH, DK, GG, GI, GK, DL, CI, AA, DN, HP, DO, DR Chameleon: CV, SY, TW, VW

Corona Austrina: $\mathrm{S}, \mathrm{VV}, \mathrm{Wa} / 1, \mathrm{Wa} / 2, \mathrm{Wa} / 3$

Other: RW Aur, GW Ori, R Mon, LH $\alpha$ 332-17, Sz 68,

GW Lup, GQ Lup, EX Lup, SR 4, SR 12, SR 9, V853 Oph,

Haro 1-16, IX Oph, KK Oph, LkH $\alpha$ 118, DI Cep, BM And, TW Hya

WTTS

Taurus: DI, UX-A, HP/G2, V 773, V819, V827, V826, V830, V836

Orion: CO, V370, KM, KN

Other: SU Aur, AB Dor, AK Sco, SZ Cha, T Cha, ROX 3, DoAr 21, DoAr 51

Data for these stars are taken from the references marked with an asterisk.
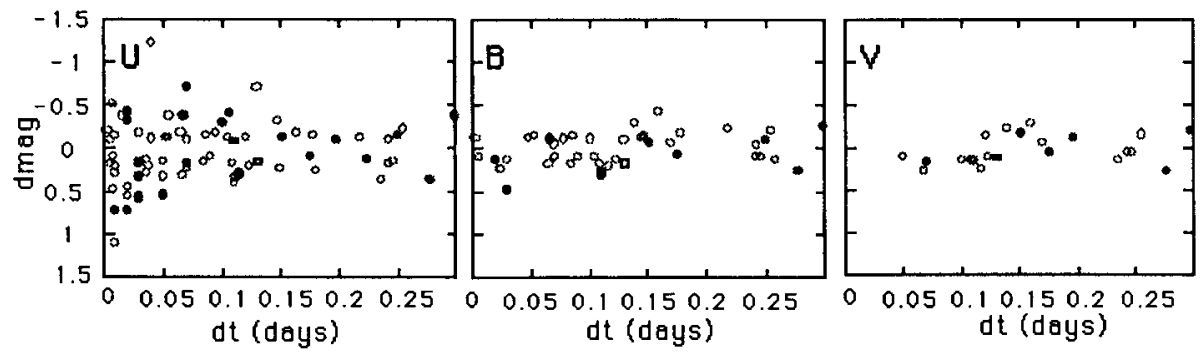

Fig. 1. Rises (d(mag.) negative) and declines with total amplitudes larger than $00^{\mathrm{m}} 05$ in $U, B$ and $V$ versus total duration, as compiled from the literature

Table 2 (designations from Herbig \& Bell 1988: HBC), and ordered according to increasing HBC number). We follow strictly the definition of an WTTS as having an equivalent width of the $\mathrm{H} \alpha$ emission line of $\mathrm{W}(\alpha) \leq 10 \AA$, although some of these stars are known to carry circumstellar dust. Only series of observations with $\geq 2$ photometric observations per night including the U-band enter. This compilation, which is based on the references marked with an asterisk in the reference list, covers a total of about 2000 hours of monitoring.

For the statistical analysis we proceed as before by measuring $d$ (magnitude), the total change in any filter over an observed time interval $d t$. If the direction of the change is the same over several measured points, then the total interval in magnitude and time is stored. Slow but significant changes can be missed by automatic procedures due to noise and we also check all nights with several observed points to restore all gross trends during a night.

Fig. 1 shows all recorded changes with $|d(\mathrm{mag})| \geq$.0.05 in $U, B$ and $V$ where the material has been divided into CTTS (subgroups: spectral types F\&G and K\&M) and WTTS. It should be noted that depending on telescope and observing 
conditions some of the points of low amplitude may be spurious. The diagrams show that: A. Rapid changes of 0.2 ( $\sim 20 \%$ in flux) or more are exceptional even in the $U$-band. B. Rapid increases (upwards in the diagrams) do not dominate over rapid declines, which would be the case if the fluctuations were flare-like. In this respect there is not a significant difference between the CTTS and WTTS as was found from a smaller sample of Strömgren photometric observations by Gahm (1990b); C. In spite of the long total patrole time, no changes larger 0.4 in $\mathrm{V}$ and only two $>0.8$ in $U$ have occurred and since eruptions with amplitudes of much more than $1^{\mathrm{m}}$. has been reported from earlier photographic work we can conclude that such variations, if real, must be extremely rare indeed. Along the same lines as before we have also derived the frequencies of rapid fluctuations, that is the probability that an observer a given night will find the star to undergo a major and rapid change ( $t \leq 0.1$ day). Regarding all changes with $|d U| \geq 0.1$ as real, we find that for the CTTS (type K\&M) this happens only $3 \%$ of the time and for the F\&G stars no event was recorded, which can be explained mainly as a contrast difference in the background $U$-flux between the two subgroups. The corresponding frequency for the WTTS is $2 \%$.

The data bank is based on published individual measurements, but some major events have only been shown as figures in the literature. This applies to the observations made in Sonneberg referred to above and also events shown by e.g. Rydgren \& Vrba (1983: on FK X-2 = V827 Tau) and Zaitseva et al. (1985: on RY Tau). Strong $U$-band flaring on two WTTS during minima in the spot modulated light-curve, was inferred from single (not time-resolved) observations by Vrba et al. (1993). Not included in the data bank is a recent, simultaneous spectroscopic and photometric study of short-term variability on 2 CTTS, 3 WTTS and one PTTS candidate by Gahm et al. (1995a). The general results from the statistical study above are confirmed. Moreover it was possible to show that all the rapid events on VW Cha (CTTS) were due to variable veiling. However, on the WTTS most events were truly flare-like, starting with a sudden increase in the Balmer line- and continuous emission. It was possible to confirm the postulate by Götz \& Wenzel (1967) about small changes in $B$ due to changes in the Balmer line fluxes. Also slower changes due to variable extinction appear to be present. Accounting for the larger radii of TTS compared to flare stars, one can conclude that the WTTS possess a magnetic surface activity comparable to the flare stars (as overviewed by Gershberg \& Shakhovskaya 1983), while there is not a single flare-like event recorded for the CTTS.

Also the profiles of emission lines can vary on short time-scales. For a review on what has been accomplished from spectroscopy at high spectral resolution see Gahm et al. (1995b).

\section{Concluding remarks}

The work in Sonneberg has been pioneering in pointing out the different components of optical variability in TTS. It is now evident that the TTS are not exceedingly active on time-scales of hours. Even at detection levels of milli-magnitudes 
the stars are mostly constant in brightness or vary only very slowly. Regarding the rapid fluctuations, some appear to be connected to exceptional magnetic surface activity - especially on the WTTS - but most of the CTTS activity comes from variable veiling, which according to current models is a consequence of mass accretion from a disk to the star, possibly along the dipole stellar magnetic field. Hence, inhomogeneous mass accretion controls the optical activity, something apparent also from night-to-night studies by e.g. Basri \& Bathala (1990), Hartigan et al. (1991) and Valenti et al. (1993). In addition, Guenther \& Hessman (1993) found accompanying variable, inverse P Cygni emission line profiles on DR Tau (CTTS). A considerable fraction of the stellar surface of a CTTS can be hidden by foreground dust and rapid infall may inhibit normal surface activity in another region around the visible pole. A possibility is that the lack of surface flaring on CTTS is due to the combination of these effects. Also, if the suggestion by Bouvier et al. (1994) and Edwards et al. (1993) is correct, namely that the CTTS rotate slower than the WTTS, then the smaller dynamo effect on the CTTS could lead to a smaller surface activity on these stars compared to the WTTS. The fact that a considerably larger fraction WTTS are detected at X-rays, compared to the CTTS, renders support to this idea (Neuhäuser et al. 1995).

However, soft X-ray events can be produced at the poles. Many X-ray flares indicate plasma temperatures which are larger than those expected from infall, and particularly on the WTTS powerful magnetic activity, sometimes very extended, is present with radio characteristics typical for flares. Simultaneous $\mathrm{X}$-ray, optical and radio monitoring provides an important tool in the diagnosis of TTS eruptions.

Acknowledgement: My thanks go to Erik Gullbring for stimulating discussions and help with the analysis of the data bank.

\section{References}

Abuladze O., Vardanian R.A., Kovalenko V.M., Kumsishvili Ja., Melikian N.D., Mironov A.V., Oshchepkov V.A., Stepanian J.A., Totochava A., Cherepashchuk A.M., Shanin G.I., Shpychka I.V., Shcherbakov A.G., 1975, Variable Stars 20, 47*

Alcala J.M., Covino E., Franchini M., Krautter J., Terranegra L., Wichmann R., 1993, A\&A 272, 225*

Appenzeller I., Mundt R., 1989, A\&AR 1, 291

Basri G., Batalha C., 1990, ApJ 363, 654

Bastian U., Mundt R., 1979, A\&AS 36, 57*

Bertout C., 1989, ARA\&A 27, 351

Bertout C., Basri G., Bouvier J., 1988, ApJ 330, 350

Bieging J.H., Cohen M., Schwartz P.R., 1984, ApJ 282, 699

Bouvier J., Bertout C., Bouchet P., 1988, A\&AS 75, 1*

Bouvier J., Cabrit S., Fernández M., Martin E.L., Matthews J.M., 1993, A\&A 272, 176

Camenzind M., 1990, in Reviews in Modern Astron. 3, G. Klare (ed.), p. 234

Cohen M., Schwartz R.D., 1976, MNRAS 174, 137* 
Covino E., Terranegra L., Franchini M., Chavarria-K C., Stalio R., 1992, A\&AS 94, 273*

Edwards S., Strom S.E., Hartigan P., Strom K.M., Hillenbrand A., Herbst W., Attridge J., Merrill K.M., Probst R., Gatley I., 1993, AJ 106, 372

Evans L., 1987, South African Astron. Obs. Circ. No 11, p. 73*

Evans L., Koen M.C.J., 1987, South African Astron. Obs Circ. No 11, p. 21*

Feigelson E.D., Giampapa M.S., Vrba F.J., 1991, in The Sun in Time, C.P. Sonett, M.S. Giampapa M.S. Matthews (eds.), Univ. of Arizona Press, p. 658

Feigelson E.D., Casanova S., Montmerle T.,Guibert J., 1993, ApJ 416, 647

Feigelson E.D., Welty A.D., Imhoff C.I., Hall J.C., Etzel P.B., Phillips R.B., Lonsdale C.J., 1994, ApJ 432, 373

Fürtig W., Wenzel W., 1964, Sterne 40, 35

Gagné M., Caillault J.-P., 1995, ApJS, in press

Gahm G.F., 1990a, in Formation of Stars and Planets and the Evolution of the Solar System, B. Battrick (ed.), ESA SP-315, p. 43

Gahm G.F., 1990b, in Flare Stars in Star Clusters, Associations and the Solar Vicinity, IAU Sym. 137, L.V. Mirzoyan, B.R. Pettersen, M.K. Tsvetkov (eds.), Kluwer, Dordrecht, p. 193

Gahm G.F., Gullbring E., Fischerström C., Lindroos K.P., Lodén K., 1993, A\&AS 100, $371^{*}$

Gahm G.F., Lodén K., Gullbring E., Hartstein D., 1995a, A\&A, in press

Gahm G.F., Petrov P., Tuominen I., Gullbring E., Iyin I., 1994b, in Proc. 4th MUSICOS Workshop, Multi-site Continuous Spectroscopy, Beijing June 1994, in press

Gershberg R.E., Shakhovskaya N.I., 1983, Ap\&SS 95, 235

Götz W., Wenzel W., 1967, Mitteilungen über Veränderliche Sterne 4, 71

Grinin V.P., Efimov Ju.S., Krasnobatsev V.I., Shakovskaya N.I., Shcherbakov A.G., Zaitseva G.V., Kolotilov E.A., Shanin G.I., Kiselev N.N., Gjulaliev Ch.G., Salmanov I.R., 1980, Variable Stars 21, 247*

Guenther E., Hessman F.V., 1993, A\&A 268, 192

Gullbring E., 1994, A\&A 287, 131

Hartigan P., Kenyon S.J., Hartmann L., Strom S.E., Edwards S., Welty A.D., Stauffer J., 1991, ApJ 382, 617

Herbig, G.F., Goodrich, R.W. 1986, ApJ 309, 294*

Herbig G.H., Bell K.R., 1988, Lick Obs. Bull., No 1111

Herbst W., Holtzman J.A., Klasky R.S., 1983 AJ 88, 1648*

Herbst W., Booth J.F., Koret D.L., Zajtseva G.V., Shakovskaya N.I., Vrba F.J., Covino E., Terranegra L., Vittone A., Hoff D., Kelsey L., Lines R., Barksdale W., 1987 AJ $94,137^{*}$

Herbst W., Herbst D.K., Grossman E., Weinstein D., 1994, AJ 108, 1906

Kardopolov V.I., Filipev G.K., 1985, Variable Stars 22, 103*

Kilkenney D., Whittet D.C.B., Davies J.K., Evans A., Bode M.F., Robson E.I., Banfield R.M., 1985, South African Astron. Obs. Circ. No 9, p. 55*

Kilyachkov N.N., Shevchenko V.S., 1976, AZh Lett. 2, 494*

Kolotilov E.A., 1986, AZh 63, 298*

Königl A., 1991, ApJ 370, L39

Koyama K., Asaoka I., Kuriyama T., Tawara Y., 1992, PASJ 44, L255

Kuhi L.V., Cram L.E., 1989, In: Cram L.E. \& Kuhi L.V. (eds.) FGK stars and T Tauri stars, NASA SP-502, p.99

Lindroos K.P., 1989, private comm.* 
Montmerle T., 1991, in The Physics of Star Formation and Early Stellar Evolution, NATO ASI Ser. C 392, C.J. Lada N.D. Kylafis (eds.), p. 675

Montmerle T., André P., 1988, in Formation and Evolution of Low Mass Stars, NATO ASI Ser. C 241, A.K. Dupree, M.T.V.T. (eds.), p. 225

Montmerle T., Koch-Miramond L., Falgarone E., Grindlay J.E., 1983, ApJ 269, 182

Montmerle T., Feigelson E.D., Bouvier J., André P., 1993, in Protostars and Planets III, E.H. Levy, J.I. Lunine (eds.), Univ. of Arizona Press, p. 68

Mundt R., 1979, A\&A 74, 21*

Neuhäuser R., Sterzik M.F., Schmitt J.H.M.M., Wichmann R., Krautter J., 1995, A\&A, in press

O'Neal D., Feigelson E.D., Mathieu R.D., Myers P.C., 1990, AJ 100, 1610

Pakull M.W., 1981, A\&A 104, 33

Panagia N., Felli M., 1975, A\&A 39, 1

Phillips R.B., Lonsdale C.J., Feigelson E.D., 1991, ApJ 382, 261

Phillips R.B., Lonsdale C.J., Feigelson E.D., 1993, ApJ 403, L 43

Preibisch Th., Zinnecker H., Schmitt J.H.M.M., 1993, A\&A 279, L 33

Pugach A.F., 1975, Variable Stars S. 2, 195*

Rucinski S.M., 1983, A\&AS 52, 281*

Rucinski S.M., 1988, Inf. Bull. Variable Stars (IAU), No $3146^{*}$

Rucinski S.M., Krautter J., 1983, A\&A 121, 217*

Rydgren A.E., Vrba F.J., 1983, ApJ 267, 191*

Rydgren A.E., Zak D.S., Vrba F.J., Chugainov P.F., Zaitseva G.V., 1984, AJ 89, 1015*

Shaimeva A.F., Shutiomova N., 1985, Variable Stars 22, 176*

Shevchenko V.S., Shutiomova N.A., 1981, Afz 17, 286*

Simon T.,Vrba, F.J., Herbst W., 1990, AJ 100, 1957*

Skinner S.L., 1993, ApJ 408, 660

Stine P.C., Feigelson E.D., André P., Montmerle T., 1988, AJ 96, 1394

Tagliaferri G., Giommi P., Angelini L., Osborne J.P., Pallavicini P., 1988, ApJ 331, L 113

Ushida Y., Shibata K., 1984, PASJ 36, 105

Ushida Y., Shibata K., 1985, PASJ 37, 515

Valenti J.A., Basri G., Johns C.M., 1993, AJ 106, 2024

Vrba F.J., Rydgren A.E., Chugainov, P.F., Shakhovskaya N.I., Zak D.S., 1986, ApJ 306, 199*

Vrba F.J., Herbst W., Booth J.F., 1988, AJ 96, 1032*

Vrba F.J., Rydgren A.E., Chugainov P.F., Shakovskaya N.I., Weaver W.B., 1989, AJ 97, 483*

Vrba F.J., Chugainov P.F., Weaver Wm.B., Stauffer J.S., 1993, AJ 106, 1608*

White S.M., Pallavicini R., Kundu M.R., 1992, A\&A 259, 149

Zaitseva G.V., 1978, Afz 14, 17*

Zaitseva G.V., 1982, Afz 18, 67*

Zaitseva G.V.,1988, Afz 31, 489*

Zaitseva G.V., Lyuti V.M., 1976, Variable Stars 20, 255*

Zaitseva G.V., Lyuti V.M., 1976, Variable Stars 20, 266*

Zaitseva G.V., Kolotilov E.A., Petrov P.P., Tarasov A.E., Shenavrin V.I., Shcherbakov A.G., 1985, AZh Lett. 11, 271 
H. Zinnecker: The missing optical surface flares on classical T Tauri stars which you emphasized suggest that these objects differ fundamentally (maybe even genetically) from the weak-line $\mathrm{T}$ Tauri stars in their magnetic field properties; or do you see another explanation?

G. Gahm: Yes, and we can only speculate on its course. Maybe the strong dipole filed connecting the disk and the star could have the effect of surpressing the development of at least very strong surface magnetic flares.

P.B. Byrne: Why, if $\mathrm{T}$ Tauri stars have deep convective zones, is the proposed dipole field not shredded and locally intensified as in the sun and, presumably, active late type stars?

G. Gahm: This is a difficult question and I have no input to give on this topic. P.B. Byrne: Is it not possible that the CTTS are more slowly rotating and so possess overall weaker fields than the active PTO?

G. Gahm: I think this postulate is very good and should be followed up. 